\title{
Evaluation of the efficacy of triphala mouth rinse as coadjuvant in the treatment of chronic generalized periodontitis: a randomized clinical trial
}

\author{
Mohammed Irfan ${ }^{1 *}$, Santosh Kumar², Viraj Amin ${ }^{3}$, Carlos Enrique Cuevas-Suárez ${ }^{1,4}$ \\ ${ }^{1}$ Federal University of Pelotas. Pelotas, Brazil \\ ${ }^{2}$ Karnavati School of Dentistry, Uvarsad. Gujarat, India \\ ${ }^{3}$ Post graduate student. Department of Periodontology, Karnavati School of Dentistry, 907/A, Uvarsad, Gandhinagar, Gujarat, India \\ ${ }^{4}$ Academic Area of Dentistry. Autonomous University of Hidalgo State. San Agustín Tlaxiaca, México
}

\begin{abstract}
Purpose: The aim of this study is to evaluate the efficacy of triphala and chlorhexidine mouthwash as coadjuvants in the treatment of chronic generalized periodontitis.

Methods: The study included 50 subjects diagnosed with chronic generalized periodontitis. After scaling and root planning treatment, patients were equally divided into 2 groups. Group A patients were prescribed with triphala mouthwash and Group B patients were prescribed with $0.2 \%$ chlorhexidine mouthwash. The clinical parameters such as the Gingival Index (GI) and Plaque Index (PI) were recorded at baseline, 7, 30 and 45 days respectively.

Results: Both treatments showed significant reductions of GI and PI after 45 days. Triphala mouthwash was better in controlling gingival inflammation (1.38 for Triphala and 1.58 for Chlorhexidine after 45 days). Both groups were similar in controlling plaque accumulation (1.59 for Triphala and 1.57 for Chlorhexidine after 45 days). Complains of metallic taste were observed in CHX group.
\end{abstract}

Conclusions: Triphala mouthwash has proven to be as effective as chlorhexidine mouthwash in chronic generalized periodontitis patients.

\section{Introduction}

Gingival and periodontal diseases, in their various forms, have afflicted humans since the dawn of history. Our understanding of the etiology and pathogenesis of oral diseases and conditions is continually changing with increased scientific knowledge. The periodontium is composed of the investing and supporting tissues of the tooth namely gingiva, periodontal ligament, cementum and alveolar bone. Periodontitis is defined as "an inflammatory disease of the supporting tissues of the teeth caused by specific microorganisms or specific group of microorganisms, resulting in the progressive destruction of the periodontal ligament and alveolar bone with pocket formation and recession or both"[1]. For treatment of periodontal disease, mechanical and chemical supragingival plaque control is essential [2].

Dental plaque has been defined as the microbial community that develops on the tooth surface, embedded in a matrix of polymers of bacterial and salivary origin [3]. Maintenance of effective plaque control is the cornerstone of any attempt to prevent and control periodontal disease. A combination of oral hygiene instructions and mechanical professional tooth cleaning at proper intervals can almost completely prevent the development of both gingivitis and periodontitis. Plaque control normally means preventive measures aimed at removing dental plaque and preventing it from recurring. This can be accomplished either mechanically or chemically [4].

Dynamic participation by an individual has to be done for proper mechanical plaque control. Therefore, the technique which is simple and less time consuming should be recommended for removing dental plaque [5]. Limitation of mechanical plaque control procedures is that they concentrate solely on the hard surfaces of the oral cavity. Although the non-shedding surfaces of the teeth provide an excellent surface for the establishment and growth of biofilms, they represent a relatively small percentage of the total area of the oral cavity (21-23\%) [6]. Chemical antiplaque agents present in mouth rinses or dentifrices could reach these soft tissue surfaces, improving the control of biofilm growth on these surfaces and delaying microbial accumulation on teeth [7].

In the developing countries of the world the widespread use of mouthwashes as an aid to oral hygiene is a relatively recent phenomenon. Chemical supra-gingival plaque control has been an issue of research for 4-5 decades. Antimicrobial agents which can prevent bacterial proliferation are developed. A great number of antimicrobial agents have been studied in regard to the control of plaque. They can be categorized into- Bisbiguanides, quaternary ammonium compounds, phenolic agents, oxygenating agents, natural products etc. From all the mouthwashes available, Chlorhexidine (CHX), a cationic bisbiguanide, is said to be a gold standard [8]. It has a promising substantivity

Correspondence to: Mohammed Irfan, Federal University of Pelotas. Rua Gonçalves Chaves, 457. Centro. CEP: 96015-560. Pelotas, Brazil, Tel/Fax: +55533225.6741; E-mail: irfan_dentart@yahoo.com

Key words: Chlorhexidine, periodontitis, triphala, mouthwash

Received: October 04, 2017; Accepted: October 24, 2017; Published: October 27,2017 
and broad-spectrum antibacterial activity [9]. The need for frequent application of CHX and side effects such as staining of teeth and unpleasant taste, has encouraged search for alternatives which are more appropriate.

"TRIPHALA" meaning "three fruits", is a traditional ayurvedic herbal formulation consisting of three fruits namely Amalaki (Emblica officinalis), Bibhitaki (Terminalia belerica) and Haritaki (Terminalia chebula) [10] combined in equal quantity. Triphala has been described in ancient Ayurvedic text as a Tridoshik Rasayana, a therapeutic agent with balancing, laxative, hemostatic, anti-inflammatory, analgesic and wound healing properties as described in sushruta samhita. Triphala is having an antiplaque efficacy which is alike to CHX. Triphala also inhibits plaque formation with minor or no side effects [11].

The purpose of the present study was to compare the efficacy of the "triphala" mouthwash over $0.2 \%$ chlorhexidine mouthwash as coadjuvant in the treatment of chronic generalized periodontitis.

\section{Materials and methods}

\section{Study design}

A clinical trial was conducted from the out-patient department of the hospital. The ethical clearance was obtained from the ethical committee of Karnavati School of Dentistry and Hospital. The participants were clearly informed about the study and a written informed consent was obtained. 50 subjects with chronic generalized periodontitis (CGP) according to AAP 1999 classification were included. CGP was defined as "clinical attachment loss $>4-5 \mathrm{~mm}$, periodontal pockets of 5-7 mm in minimum 3 teeth of each quadrant". Subject with chlorhexidine allergy were excluded from the study. The duration of the study was 45 days. Patients were equally divided into two groups. Group A were treated with scaling and root planning and prescribed to use triphala mouthwash. Triphala mouthwash is prepared by boiling 100 grams of Triphala powder into $1000 \mathrm{ml}$ of water and reduced to half [12]. Group $\mathrm{B}$ were treated with scaling and root planning and prescribed to use $0.2 \%$ chlorhexidine as mouthwash. Patients were allotted to group A and $\mathrm{B}$ on the basis of lottery system.

Patient were asked to rinse their mouth with $10 \mathrm{~mL}$ of the allotted mouthwash for 1 minute twice daily for 45 days. Patients were educated with the oral hygiene instructions. The plaque index (PI: Quigley and Hein Plaque Index) and the gingival index (GI: Loe and Silness Plaque Index) [13] were assessed in each patient on the $7^{\text {st }}, 30^{\text {th }}$ and the $45^{\text {th }}$ day by two different skilled examiners. The efficacy outcome was examined with the changes in the GI and PI. Also, at each appointment patients were inquired about any side effect regarding the use of mouthwash.

\section{Statistics}

The statistical analysis was performed using IBM SPSS Statistics 20 Software (Armonk, NY. USA). The Student $t$ test for paired data was used to evaluate the changes after treatment within groups. An independent sample t-test was used to compare difference between groups index scores recorded at baseline, 7, 30 and 45 days.

\section{Results and discussion}

A total of 50 patients ( 32 males and 18 females) were selected for the study. All the patients completed the study. The triphala mouthwash was equally accepted and did not show any adverse reactions. After 45 days of duration, all the group individuals had similar gingival index and the plaque scores as compared to the baseline levels. The intra examiner variability calculated using Cohen's kappa statistics was $96.1 \%$.
In group A (triphala group) at baseline the mean gingival score was $1.83 \pm 0.17$ and $1.38 \pm 0.38$ after 45 days (Table 1 and Graph 1 ). Likewise, at baseline the mean plaque score was $1.78 \pm 0.20$ and 1.59 \pm 0.21 after 45 days. In group B (CHX group) at baseline the mean gingival score was $1.94 \pm 0.27$ and $1.58 \pm 0.25$ after 45 days. Likewise, at baseline the mean plaque score was $1.72 \pm 0.20$ and $1.57 \pm 0.21$ score was recorded after 45 days (Table 2 and Graph 2). Both mouthwashes had been beneficial in reducing plaque and improving gingival status.

When chlorhexidine was compared with triphala data in terms of gingival indexes, at baseline it was not statically significant but after 7, 30 and 45 days it was highly significant. Regarding to plaque index, although the results were beneficial they were not significant at any stage of study.

Oral healthcare is an essential part of general health. Foreign bodies and oral bacteria can be a threating source of aspiration pneumonia and endocarditis [14]. Therefore, herbal medicines which are naturally occurring provide most dependable and reliable results for restoration of oral and systemic health [12]. Antimicrobial mouthwashes used in periodontics have either sugar or alcohol in one of their contents [15]. These components increase the possibility of having caries and halitosis. Thus, by the using herbal mouth rinses, these side effects can be avoided [9].

The knock of Ayurveda on the doors of science is resonating with each passing day. The term Ayurveda is a compound term wherein "Ayu" denotes life and "veda" denotes knowledge. Ayurveda originated more than 3000 years ago from the youngest of vedas, "The Atharva Veda". It inspires the use of herbal medicines. The traditional indian texts, the charak Samhita and susruta Samhita describes the method for traditional herbal supplement [12]. The aptitude behind triphala's many benefits comes from individual herbs which constitute the formula.

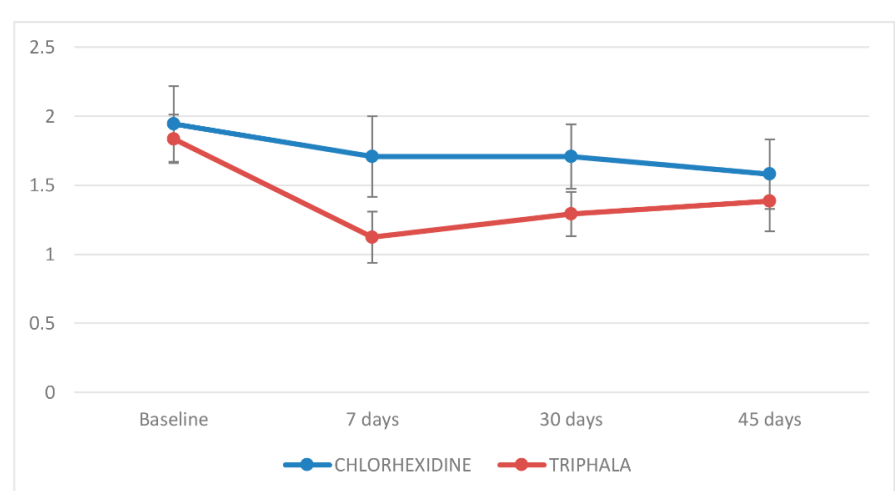

Graph 1. Comparison of Gingival index at $0,7,30$ and 45 days

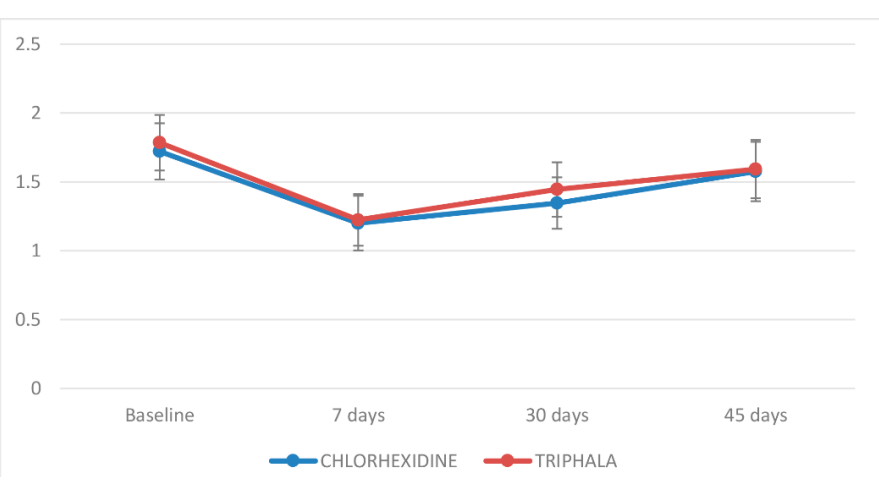

Graph 2. Comparison of plaque index at $0,7,30$ and 45 days 
Table 1. Comparison of Loe and Silness index between both the groups at baseline, $7^{\text {th }}$ day, $30^{\text {th }}$ day and $45^{\text {th }}$ Day.

\begin{tabular}{|c|c|c|c|c|c|c|c|c|}
\hline \multicolumn{9}{|c|}{ Independent $t$ test } \\
\hline & GROUP & $\mathrm{N}$ & Mean & Std. Deviation & Std. Error Mean & $\mathrm{t}$ & $\mathrm{df}$ & P value \\
\hline \multirow{2}{*}{ BASELINE } & Chlorhexidine & 25 & 1.9440 & .27398 & .05480 & \multirow{2}{*}{1.660} & \multirow{2}{*}{48} & \multirow{2}{*}{.103} \\
\hline & Triphala & 25 & 1.8360 & .17531 & .03506 & & & \\
\hline \multirow{2}{*}{ SEVEN } & Chlorhexidine & 25 & 1.7080 & .29143 & .05829 & \multirow{2}{*}{8.453} & \multirow{2}{*}{48} & \multirow{2}{*}{.000} \\
\hline & Triphala & 25 & 1.1240 & .18547 & .03709 & & & \\
\hline \multirow{2}{*}{ THIRTY } & Chlorhexidine & 25 & 1.7080 & .23438 & .04688 & \multirow{2}{*}{7.322} & \multirow{2}{*}{48} & \multirow{2}{*}{.000} \\
\hline & Triphala & 25 & 1.2920 & .16052 & .03210 & & & \\
\hline \multirow{2}{*}{ FORTYFIVE } & Chlorhexidine & 25 & 1.5800 & .25166 & .05033 & \multirow{2}{*}{2.947} & \multirow{2}{*}{48} & \multirow{2}{*}{.005} \\
\hline & Triphala & 25 & 1.3840 & .21733 & .04347 & & & \\
\hline
\end{tabular}

Table 2. Comparison of Quigley Hein plaque index between both the groups at baseline, $7^{\text {th }}$ day, $30^{\text {th }}$ day and $45^{\text {th }}$ Day.

\begin{tabular}{|c|c|c|c|c|c|c|c|c|}
\hline \multicolumn{9}{|c|}{ Group Statistics } \\
\hline & GROUP & $\mathrm{N}$ & Mean & Std. Deviation & Std. Error Mean & $\mathrm{t}$ & $\mathrm{df}$ & $P$ value \\
\hline \multirow{2}{*}{ BASELINE } & Chlorhexidine & 24 & 1.7208 & .20426 & .04169 & \multirow{2}{*}{-1.090} & \multirow{2}{*}{48} & \multirow{2}{*}{.281} \\
\hline & Triphala & 25 & 1.7840 & .20141 & .04028 & & & \\
\hline \multirow{2}{*}{ SEVEN } & Chlorhexidine & 24 & 1.2000 & .19781 & .04038 & \multirow{2}{*}{-.436} & \multirow{2}{*}{48} & \multirow{2}{*}{.665} \\
\hline & Triphala & 25 & 1.2240 & .18771 & .03754 & & & \\
\hline \multirow{2}{*}{ THIRTY } & Chlorhexidine & 24 & 1.3458 & .18645 & .03806 & \multirow{2}{*}{-1.785} & \multirow{2}{*}{48} & \multirow{2}{*}{.081} \\
\hline & Triphala & 25 & 1.4440 & .19807 & .03961 & & & \\
\hline \multirow{2}{*}{ FORTYFIVE } & Chlorhexidine & 24 & 1.5750 & .21518 & .04392 & \multirow{2}{*}{-.279} & \multirow{2}{*}{48} & \multirow{2}{*}{.782} \\
\hline & Triphala & 25 & 1.5920 & .21197 & .04239 & & & \\
\hline
\end{tabular}

Triphala has been extensively used in Ayurveda because of its various properties and therapeutic uses. The original meaning of word triphala is "three fruits" derived from haritaki, bibhitaki and vibhitaki. Triphala helps in detoxification of the colon and purifies the blood of the whole body with removal of the toxins from the liver. Triphala also has added benefits of reduction of serum cholesterol and high blood pressure. Triphala can also bring relief to the stomach-related problems like decreased appetite, stomach acidity, abdominal pain and constipation. Furthermore, this peculiar herb is utterly effective in treatment of common cold and cough. The contents of triphala such as phenols, tannins and glycosides are responsible for its potent antioxidant activity [16].

Periodontal disease can be managed by herbal extracts that are potent inhibitors of pathologically elevated collagenases. Lee et al concluded that connective tissue breaks down during inflammatory periodontal disease, this mechanism is mediated by polymorphonuclear neutrophils which provide the major source of collagenase or gelatinase. Allan et al concluded that activity of MMP-9 can be inhibited by triphala. Triphala is also known to cause suppression of collagenase activitys [16].

The triphala mouthwash is easy to use and considering the fact that subjects of the current study reported brown staining and complained of bitter taste with CHX, it is advisable that clinicians can safely prescribe the triphala mouthwash to their patients. This being a shortterm study, the results can be used as a baseline data for future studies with similar study design.

In this study Gingival index (Loe and Silness) showed a statically significant difference between group A and group B. Triphala showed a better healing of gingiva than chlorhexidine. This can be attributed to triphala's antibacterial activity against methicillin resistant staphylococcus Aureus (MRSA) [17]. Triphala is rich in polyphenols and ascorbic acid. These polyphenols are considered to be antimicrobial agents. Besides triphala has also shown the presence of epigallocatechin gallate (EGCG) as one of the condensed tannins [18]. This EGCG binds to the cell wall of bacteria and stop the biosynthesis thus inhibiting the bacterial growth, promoting an inhibitory action towards proteases and collagenase [19]. Hence it is instrumental in promoting synthesis of collagen by preventing degradation of collagen by the above enzymes. One of the most active agent in triphala is ascorbic acid which enhances collagen synthesis at any inflamed site. These above properties of triphala might be responsible for a better healing of gingiva when compared to chlorhexidine.

Our study also showed that there was no statically significant difference in relation to plaque index and papillary marginal index. This can be attributed to patient's oral hygiene practices. Although all the patients were instructed to maintain oral hygiene habits but there is always a chance of not maintaining. The mean value of plaque index showed that there were improvements in both the groups but not statically significant. In CHX group some patients complained of bad taste whereas no similar complaints were in triphala group. Surprisingly many of them had a good feedback regarding the taste and its herbal nature.

This asserted that triphala mouthwash when compared to $0.2 \%$ chlorhexidine was better in reducing inflammation and healing gingiva but at the same time both were similar in controlling the plaque. Since triphala mouthwash is of herbal origin, better in reducing the gingival condition and also there is no chances of bacterial resistance, so it should be considered as best alternative to chlorhexidine.

\section{Conclusion}

Within the limits of the study, it was concluded that the triphala and chlorhexidine mouthwash were effective in reducing the gingival and plaque index but triphala was more effective in reducing gingival inflammation. Moreover, triphala have less chance of developing bacterial resistance. Hence it should be considered as alternative to chlorhexidine.

\section{Conflict of interest}

No potential conflict of interest relevant to this article was reported. 


\section{References}

1. Newman MG, Carranza F, Takei HH, Klokkevold PR. Clinical Periodontology. 10th ed. Saunders Elsevier; 2006

2. Mendez M, Melchiors Angst PD, Stadler A, Oppermann R, Gomes S (2016) Impacts of supragingival and subgingival periodontal treatments on oral health-related quality of life. Int J Dent Hyg. 15:135-141 [Crossref]

3. Marsh PD, Martin M V, Lewis MAO, Williams D. Oral Microbiology. 5th ed. Edinburgh: Elsevier Health Sciences UK; 2009.

4. Axelsson P. Concept and practice of plaque-control. Pediatr Dent. 1981;1:101-13.

5. Löe H, Kleinman D V (1986) Dental plaque control measures and oral hygiene practices: proceedings from a state-of-the-science workshop. Washington: Oxford University Press, USA.

6. Kerr WJ, Kelly J, Geddes DA (1991) The areas of various surfaces in the human mouth from nine years to adulthood. $J$ Dent Res 70: 1528-1530. [Crossref]

7. Teles RP, Teles FRF (2009) Antimicrobial agents used in the control of periodontal biofilms: effective adjuncts to mechanical plaque control? Braz Oral Res. $23 \mathrm{Suppl}$ 1:39-48. [Crossref]

8. Mathur S, Mathur T, Srivastava R, Khatri R (2011) Chlorhexidine: The gold standard in chemical plaque control. Natl J Physiol Pharm Pharmacol. 1:45-50.

9. Nagappan N, John J (2012) Antimicrobial Efficacy of Herbal and Chlorhexidine Mouth rinse - A systematic review. J Dent Med Sci. 2:5-10.

10. Bhavikatti SK, Dhamija R, Prabhuji ML V (2015) Triphala: Envisioning its role in dentistry. Int Res J Pharm. 6:309-13.
11. Narayan A, Mendon C (2012) Comparing the effect of different mouthrinses on de novo plaque formation. J Contemp Dent Pract 13: 460-463. [Crossref]

12. Malhotra R, Grover V, Kapoor A, Saxena D (2011) Comparison of the effectiveness of a commercially available herbal mouthrinse with chlorhexidine gluconate at the clinical and patient level. J Indian Soc Periodontol. 15:349-352. [Crossref]

13. Rebelo MAB, Queiroz AC de. Gingival Indices: State of Art, Gingival Diseases - Their Aetiology, Prevention and Treatment. In: Panagakos F, editor. Gingival Diseases - Their Aetiology, Prevention and Treatment. INTECH Open Access Publisher; 2011. p. 246.

14. Griffiths J, Jones V, Leeman I, Lewis D, Patel K, et al. (2000) Guidelines for the Development of Local Standards of Oral Health Care for Dependent, Dysphagic, Critically and Terminally Ill Patients. London: British Society for Disability and Oral Health; 2000. (BSDH Guidelines: Unlocking barriers to care).

15. Leyes Borrajo JL, Garcia VL, Lopez CG, Rodriguez-Nuñez I, Garcia FM, et al. (2013) Efficacy of chlorhexidine mouthrinses with and without alcohol. Dentistry Today. $317-21$.

16. Gupta M (2010) Therapeutic uses of the polyherbal drug Triphala in geriatric diseases Int J Pharma Bio Sci. 1(2).

17. Kirubanandan S, Swethkamal K, Renganathan S (2013) Activities of triphala towards promoting collagen synthesis at wound site and inhibiting methicillin-resistant Staphylococcus aureus and its enzymes. Int J Pharm Pharm Sci. 5:54-62.

18. Kumar MS, Kirubanandan S, Sripriya R, Sehgal PK (2008) Triphala Promotes Healing of Infected Full-Thickness Dermal Wound. J Surg Res. 144:94-101. [Crossref]

19. Cuesta AI, Jewtuchowicz VM, Brusca MI, Mujica MT, Rosa AC (2011) Antibiotic susceptibility of Staphylococcus aureus isolates in oral mucosa and pockets of patients with gingivitis-periodontitis. Acta Odontol Latinoam. 24:35-40. [Crossref] 
Irfan M (2017) Evaluation of the efficacy of triphala mouth rinse as coadjuvant in the treatment of chronic generalized periodontitis: a randomized clinical trial 
Irfan M (2017) Evaluation of the efficacy of triphala mouth rinse as coadjuvant in the treatment of chronic generalized periodontitis: a randomized clinical trial

Copyright: ( 02017 Irfan M. This is an open-access article distributed under the terms of the Creative Commons Attribution License, which permits unrestricted use, distribution, and reproduction in any medium, provided the original author and source are credited. 